\title{
Limited Sinogram Completion for Transmission SPECT Imaging
}

\author{
Cyril Riddell, W. Craig Barker, Stephen L. Bacharach \\ National Institutes of Health, Bldg. 10 Room 1C401, Bethesda, MD 20892
}

\begin{abstract}
Truncation artifacts can occur in simultaneous emission transmission SPECT imaging even with parallel geometry, especially when the acquisition geometry is optimized for emission at the expense of the transmission data. We isypothesized that the addition of only a few projections sampling truncated areas (by shifting the bed/camera) would permit a significant improvement in image quality with only a small increase in imaging time. In parallel geometry, data are preprocessed and the additional projections are merged into the original sinogram, thereby partially completing it. For fan-beam data, the projector routine is modified to take into account different bed positions. Both cases require the use of an iterative reconstruction algorithm. Improvements due to partial completion are shown for different increases of total imaging time for both geometries. On simulated and true data, a $15 \%$ increase of imaging time led to a better recovery of the truncated area than obtained from a priori knowledge of the body contour.
\end{abstract}

\section{INTRODUCTION}

Multi-headed SPECT cameras equipped with line sources can simultaneously acquire both transmission and emission data $[1,2]$. One popular configuration allows parallel geometry, using $90^{\circ}$ dual-headed cameras with scanning line sources. Precise bed motion is used to position the patient as close as possible to both collimators for optimal resolution at each step. Such positioning, while optimal for resolution, will frequently result in truncation. Depending on the distribution of the radioisotope, this truncation is often of no consequence for the emission scan, but may greatly affect the attenuation map, and therefore produce incorrect attenuation compensation. Transmission data can also be acquired with three-headed gamma-cameras by use of a line source fixed at the focal point of a fan-beam collimator. Fixed fan-beam line sources offer better statistics than scanning line sources, but the magnification inherent in fan-beam collimators makes truncation of the attenuation data inevitable. The emission data can be acquired truncation free by using parallel collimators on the two other heads [3].
Iterative reconstruction techniques substantially limit the impact of truncation error with respect to the filtered backprojection algorithm (FBP), allowing improved lesion detection with attenuation compensation in cardiac imaging $[3,4]$. To overcome truncation, additional information can be gathered. Assumed or measured body contours have been used for analytically completing the truncated projections [5] or for improving iterative reconstruction performance [6-10]. However, a method to make an accurate estimation of the body contour is not available on most current generation gamma-cameras. In fan-beam geometry, it has been shown that truncation could be prevented by scanning the subject at two different bed positions [11] or with an off-centered fan-beam collimator [12]. However, successive, rather than simultaneous, acquisitions were necessary.

We describe here a method which in effect reduces transmission truncation for parallel geometry. This method makes use of bed motion, which is a feature of most dualheaded cameras. The method involves positioning the bed for optimal emission imaging, followed by translation of the bed to permit resampling of truncated transmission areas at only a few additional projections. We describe here the application of the method to both simulated and actual measured parallel geometry SPECT data. In addition, the technique has been adapted for and applied to simulated fan-

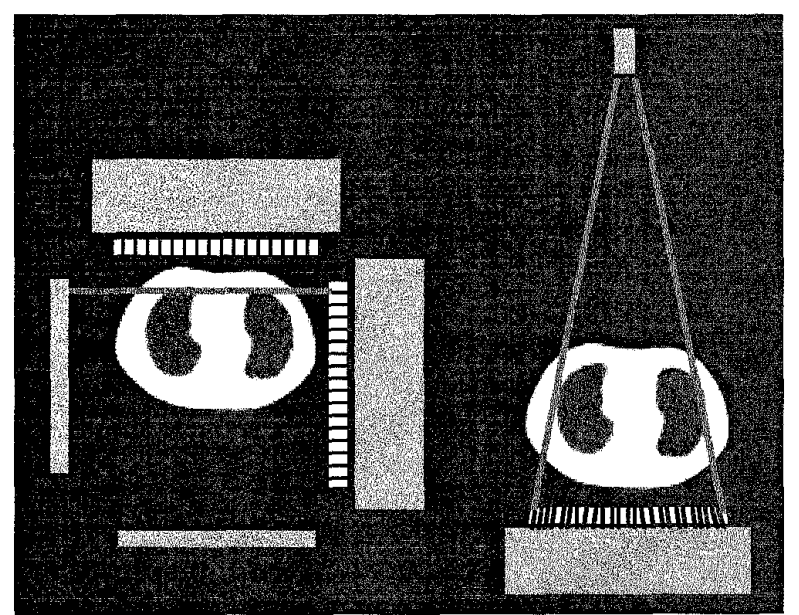

Fig. 1: Dual-headed SPECT gamma-cameras are equipped with 2 line sources (left). Truncation can occur because of the gap between the heads. Three-headed cameras have only one head for transmission data with the line source fixed at the focal point of a fan-beam collimator (right). 
beam data.

We show that even with very few additional projections, adequate restoration of the truncated image can be achieved. If successful, this scheme might be of clinical importance by permitting more accurate attenuation compensation with very little increase in imaging time.

\section{SINOGRAM "COMPLETION"}

\section{A. Parallel geometry:}

Let $(O, x, y)$ be a fixed coordinate system and $(O, u, v)$ a rotating one. Positive angles go counterclockwise with $0^{\circ}$ at 3 o'clock. At angle $\theta: u(\theta)=x \cos \theta+y \sin \theta$ and $v(\theta)=-x \sin \theta+y \cos \theta$.

We consider a transmission sinogram $s(\theta, v)$ acquired from an object in a position suitable for gathering the emission data but resulting in truncation of the transmission data. We denote $s_{x_{0}, y_{0}}(\theta, v)$ as the sinogram of the same object after it has been translated to position $x_{0}$ and $y_{0}$ in the fixed coordinate system, or $u_{0}(\theta)$ and $v_{0}(\theta)$ in the rotating system. This sinogram is related to the sinogram $s(\theta, v)$ of the non translated object $f$ by:

$$
\begin{aligned}
& s_{x_{0}, y_{0}}(\theta, v)=\int f\left(u-u_{0}, v-v_{0}\right) d\left(u-u_{0}\right) \\
& =\int f\left(u, v-v_{0}\right) d u=s\left(\theta, v-v_{0}\right)
\end{aligned}
$$

Because $s_{x_{0}, y_{0}}(\theta, v)$ can be merged with $s(\theta, v)$ by shifting each row by $-v_{0}(\theta)$, we can partially "complete" the sinogram with a limited number of additional projections. To minimize interpolation errors, the additional projections resample some of the previously acquired projections, i.e. at angular positions already sampled. Two measurements of the same projection bin are averaged together. When a translation introduces a fractional measurement at an edge of the resampled projection, the value is discarded.

\section{B. Fan-beam geometry:}

Fan-beam data can be resampled into parallel data and can then be combined as previously described. However, interpolation must be performed very carefully to avoid a loss of resolution. Alternatively, we describe our modifications to the RECLBL line-length projector routine $[13,14]$ to include multiple bed positions.

Three parameters are affected by translation in our fanbeam projector: the distance from the focal point to the center of rotation (rfan), the magnification factor ( $p w i d$ ) and the projection of the center of rotation onto the projection axis (axisu).

For each bed position, the projections are acquired with the same values for (rfan, pwid, axisu), i.e. the detector is fixed and the object changes position. During reconstruction, the object is at position $(0,0)$ and the detector is considered to move. The fan-beam parameters are updated according to the projection angle $\theta$ and the corresponding bed position $\left(u_{0}(\theta), v_{0}(\theta)\right)$ as follows:

$$
\left\{\begin{array}{l}
r f a n_{0}(\theta)=r f a n+u_{0}(\theta) \\
\text { wwid }_{0}(\theta)=\left(r f a n / r f a n_{0}(\theta)\right) p w i d \\
\operatorname{axisu}_{0}(\theta)=\text { axisu }^{2} v_{0}(\theta) \text { pwid }_{0}(\theta)
\end{array}\right.
$$

For $u_{0}(\theta)>0$, the object is closer to the detector, the distance $r f a n$ is increased, and the magnification factor pwid is decreased. For $v_{0}(\theta)>0$, the projection at the center of rotation is increased according to the magnification. Finally, the projection must be scaled by $p w i d / p w i d_{0}(\theta)$ to maintain the total sum equal for each (complete) projection.

Therefore the additional projections are no longer merged into the original sinogram, but instead are concatenated with new geometrical parameters.

\section{RECONSTRUCTION:}

\section{A. Frequency Weighted Least Squares:}

Because only a few additional projections are used, the data set is still incomplete. For incomplete data, an iterative algorithm is used, with the Radon transform matrix $R$ replaced by $R_{t r}$ whose coefficients relating a pixel $i$ to bin $j$ are defined by $\left(R_{t r}\right)_{j i}=R_{j i}$ if bin $j$ has been measured, and $\left(R_{t r}\right)_{j i}=0$ otherwise. There are several possible choices of iterative algorithms one could use, but the algorithm must be constrained or the reconstruction will not be stable. Two frequently used algorithms are the MLEM algorithm for emission [15], which includes the positivity constraint but is not linear, and the conjugate gradient algorithm, whose stability is easily ensured through regularization. We used an enhanced version of the conjugate gradient: the frequency weighted least squares algorithm (FWLSQ) [9] which includes a ramp filtering step. This step speeds the convergence up and provides full resolution at any iteration. In this particular case, it also reformulates the problem in a manner which makes it easier to set the regularization parameter. Poisson transmission noise was not included in the model, although new algorithms are available which can do so [16].

All reconstructions were performed by minimizing the following criterion with the conjugate gradient scheme ( $D$ is the operation of ramp filtering, $\alpha$ is a scalar and $\nabla$ is the gradient operator):

$$
\min _{f}\left\{\left\|D^{1 / 2}\left(R_{t r} f-s\right)\right\|^{2}+\alpha\|\nabla f\|^{2}\right\}
$$




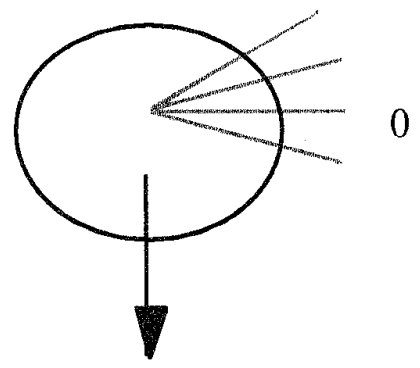

Fig. 2: Completion angles for parallel data. The bed is moved downwards and 1 to 4 projections are taken around $0^{\circ}$

\section{B. Gaussian regularization:}

Incomplete data means that the number of measurements is not sufficient to assign a unique image to the truncated sinogram. Regularization provides a unique smooth approximation of the true distribution at the expense of a loss in resolution. Linking the regularization parameter to the amount of smoothing is not trivial in general, but in this particular case can be related to gaussian filtering.

The linear system solved by (3) is the following:

$$
\left(R_{t r}^{t} D R_{t r}+\alpha \Delta\right) f=R_{t r}^{t} D s
$$

with $\Delta$ being the Laplacian operator with central value equal to 4 . When truncation tends to 0 (therefore for those pixels not affected by truncation), we have $R_{t}^{t} D R_{t r} \rightarrow I$ (the identity matrix) and $R_{t r}^{t} D s \rightarrow f^{*}$ (the perfect map), so that the solution of (4) becomes:

$$
f=(I+\alpha \Delta)^{-1} f^{*} \cong(I-\alpha \Delta) f^{*}
$$

for $\alpha$ small. It has been shown that gaussian filtering can be computed iteratively by considering it as a diffusion process over time $t$ :

$$
f^{(t+d t)}=(I-d t \Delta) f^{(t)}
$$

with $d t$ small. Applying this diffusion scheme to an image $f$ at $t=0$ results, at $t=t_{0}$, in the filtering of $f$ by a gaussian of full width at half maximum (fwhm) $\sqrt{16 \ln (2) t_{0}}[17,18]$. Therefore, for a small $\alpha$, inspection of equations (5) and (6) above show that our regularization will be analogous to a gaussian filtering of fwhm $3.33 \sqrt{\alpha}$. We set $\alpha=0.125$ (fwhm 1.2 pixel) which is small enough such that equation (5) is true, yet large enough to avoid high frequency patterns. Although this filter is sufficient to ensure stability, in the presence of significant noise levels additional filtering may be desired.

\section{Completion versus Contour:}

To evaluate the impact of our proposed completion technique, it was compared to the results obtained with a priori knowledge of the body contour. Such knowledge improves image recovery from truncated data because it reduces the number of unknown pixels by setting to 0 all those pixels of the grid that do not belong to the body. Note that in a similar way, the grid size is of importance since it defines the number of unknown pixels. Artifacts are produced if the grid is unnecessarily large with respect to the size of the object by introducing too many degrees of freedom and thereby allowing too many pixels to vary outside the object boundaries.

\section{EXPERIMENTS AND RESULTS}

\section{A. Simulation and phantom study for parallel geometry:}

A human torso phantom (Data Spectrum Inc) was scanned using two different bed positions with a $90^{\circ}$ dualheaded SPECT camera equipped with two scanning line sources (ADAC corp. Vertex scanner) and parallel hole collimation. Detectors were $40 \mathrm{~cm}$ long in the transaxial plane with a gap at their junction. Phantom dimensions were $38 \times 26 \mathrm{~cm}$. All acquisitions were performed using 60 projections over $180^{\circ}\left(3^{\circ}\right.$ increment from $-45^{\circ}$ to $\left.135^{\circ}\right)$. An acquisition matrix of $128 \times 128$ was used with a pixel size of $4.56 \mathrm{~mm}$. The first scan was truncated because the phantom was placed very close to one head (e.g. for optimized emission resolution in cardiac SPECT), and therefore was partially out of the field of view of the second head (see fig. 1). To investigate the efficacy of using additional projections to correct for this truncation, a second scan was performed with the bed shifted down $7.7 \mathrm{~cm}$ in a position that avoided truncation (at the sacrifice of resolution). The fully completed scan was reconstructed with FBP and provided a blurred estimate of the object contour.

The same imaging situation was simulated. A CT image of the phantom (shown in fig. 1) was taken at a similar slice location with the values scaled to Gadolinium153 attenuation values $\left(0.17 \mathrm{~cm}^{-1}\right.$ and $0.06 \mathrm{~cm}^{-1}$ for tissue and lung respectively). A contour was extracted after blurring the CT based image with a gaussian of fwhm 2 pixels. With the previous acquisition parameters, 60 noise free projections were generated.

To sample the region where truncation occurred (i.e. to complete the projections), 4 additional projections were acquired with the second head for both the real and the simulated phantom. The first resampled projection was at $0^{\circ}$, the second at $-15^{\circ}$, the third and fourth at $-30^{\circ}$ and $+15^{\circ}$ (see fig. 2). Since 30 angular positions are needed for a full scan with two heads, these 4 additional positions required only a $15 \%$ increase in imaging time.

The reconstruction grid size was varied by taking the circle inscribed in a $128 \times 128$, a $110 \times 110$ and a $90 \times 90$ 


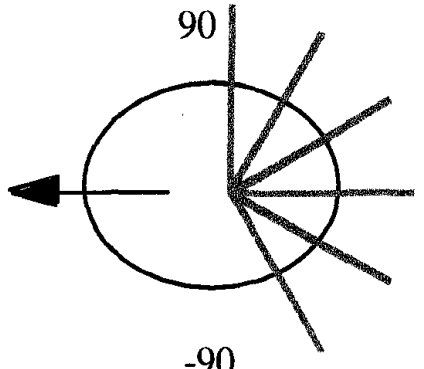

$-90$

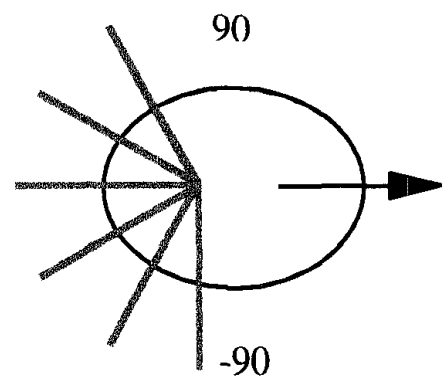

Fig. 3: Partial completion of a truncated fan-beam transmission scan with 12 projections and 2 bed positions.

square grid (with fixed pixel size of $4.56 \mathrm{~mm}$ ), and by using the a priori knowledge of the contour.

Using the extra projections described above to complete the sinogram affects not only the attenuation image, but even more importantly, the emission image. To investigate how emission quantification is affected, we simulated the acquisition (same parameters as before) of an emission distribution attenuated by the attenuation map derived from the CT image. The distribution was a uniform disk (diameter $18 \mathrm{~cm}$ ) at the center of the grid and was small enough as to not be truncated. Reconstructions with 30 iterations of the EM algorithm were performed with the different attenuation maps.

\section{B. Simulation for fan-beam geometry:}

With three-headed cameras and fan-beam geometry, we considered the same CT based attenuation image to be at the center of the reconstruction grid. The simulated fanbeam geometry had a $65 \mathrm{~cm}$ focal length. Projections were simulated with a $3^{\circ}$ increment over $360^{\circ}$, yielding 120 angular positions. The orbit was circular with a radius of rotation set at $23 \mathrm{~cm}$. The pixel size being $4.56 \mathrm{~mm}$, the following parameters were obtained in pixels: $r f a n=88$, pwid $=1.547$, axisu=40.5. A $40 \mathrm{~cm}$ detector would provide 88 projection bins but the truncation was simulated by taking only 80 bins. Thus, the truncation affected not only the external contour, but also the edges of the lung.

Additional projections were taken in the following manner: The bed was moved to the left and a $180^{\circ}$ acquisition was simulated, going clockwise and starting at $90^{\circ}$ with respectively $6,9,15$ and 30 equally spaced projections. The bed was also moved to the right and a complementary $180^{\circ}$ acquisition was simulated going clockwise starting at $-90^{\circ}$. All together, $12,18,30$ and 60 projections were added respectively, implying an increase in imaging time of $5,15,25$ and $50 \%$. This scheme is illustrated on fig. 3 for 6 projections per bed position.

All the reconstructions were performed within a $128 \times 128$ grid. The completion results were compared to using no additional information (i.e. using neither additional projections nor knowledge of the contour) and to using only knowledge of the contour.

The effect of sinogram completion on emission quantification was studied by sinnulating the untruncated, parallel collimated, acquisition of an emission distribution identical to the CT attenuation image (i.e. the emission intensity was proportional to the attenuation values). This emission distribution was then attenuated by the CT based attenuation map. Reconstructions with 30 iterations of the EM algorithm were performed with the different attenuation maps.

\section{Results:}

The convergence of the reconstruction algorithm was evaluated by computing the normalized root mean square (NRMS) error between the result for each iteration of the reconstruction process and the CT based attenuation image as the reference image ( $\| f^{(n)}-$ ref $\|/\|$ ref $\|$ ) over the known extent of the object. Fig. 4 shows the plot of this error for FWLSQ applied to parallel-beam noise free data for the three grid sizes with no additional information and when using knowledge of the contour. Fig. 5 shows the images reconstructed within the $128 \times 128,110 \times 110$ and $90 \times 90$ grids (images $5-\mathrm{A}$ to $5-\mathrm{C}$ ), and within the contour (5-D). Although going from a $128 \times 128$ grid to a $110 \times 110$ grid might seem a small change, the impact was significant on both the error after 100 iterations and the convergence rate, showing that the $128 \times 128$ grid was unnecessarily large. The $90 \times 90$ grid did not contain many background pixels (as can be seen on fig. 5) but there was still an improvement in using the body contour, more so for the convergence rate than for the final error. Images 5-A to 5-C show that the truncated area anterior to the lungs was reconstructed beyond its normal limit whereas the lungs were correctly delineated. Without additional information, the truncated region, which should be uniform, was not recovered. Artifacts were also generated outside the contour to an extent proportional to the grid size. Grid size reduction was profitable by reducing the final error computed over the object as well as the extent and magnitude of artifacts outside the object boundaries. Knowledge of the body contour avoids generating artifacts outside the object and gives accurate outer edges, but did 


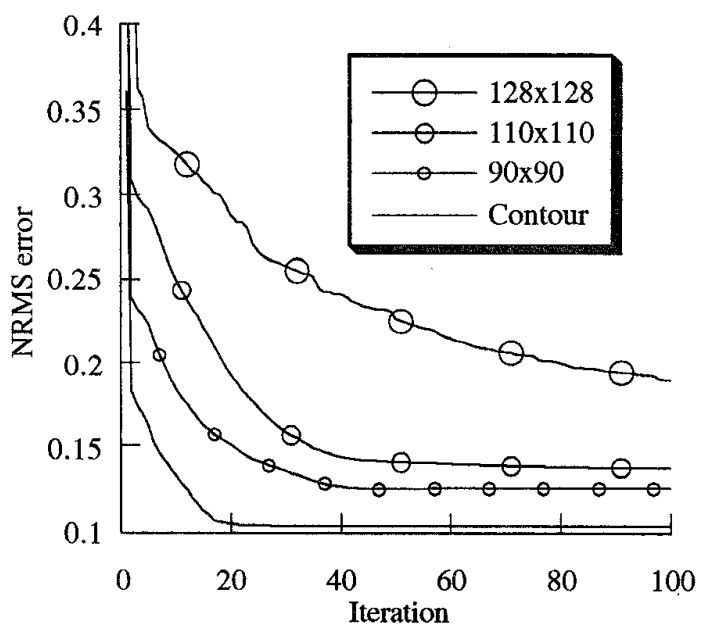

Fig. 4: Convergence of the FWLSQ algorithm for noise free parallel-beam truncated data reconstructed with no additional information and grids of size $128 \times 128,110 \times 110$ and $90 \times 90$, and with contour knowledge.

not produce uniform attenuation values in the anterior chest wall. The regularization was efficient at stabilizing the reconstruction process in all cases.

Fig. 6 shows the plot of the NRMS errors for the different numbers of additional projections using a $110 \times 110$ grid. Each additional projection provided a significant improvement. However, the body contour provided a much faster convergence. With 4 projections, the completion method outperformed the contour method inside the contour. The last curve shows that with a $128 \times 128$ grid, adding 4 projections fell short from matching the contour method.

Attenuation images reconstructed with the completion technique are shown in fig. 7 with 1 (image 7-A), 2 (7-B) and 4 (7-C) additional projections (grid 110x110) and

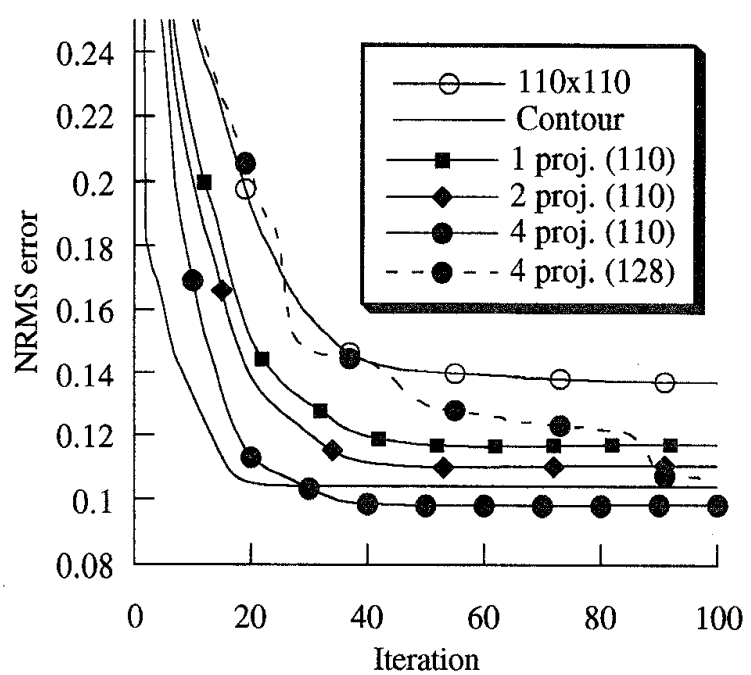

Fig. 6: Convergence of the FWLSQ algorithm for noise free parallel-beam truncated data with: no additional information and a $110 \times 110$ grid, contour knowledge, 1,2 , and 4 additional projections with a $110 \times 110$ grid, and 4 additional projections and a $128 \times 128$ grid.

compared to the contour knowledge (image 7-D). As the sinogram was completed with 1 to 4 additional projections, the artifacts outside the body decreased and the anterior chest wall was more accurately depicted. The additional projections improved uniformity in the anterior chest wall, with 4 projections giving better uniformity in this region than was obtained with knowledge of the contour. Both methods, however, yielded a subtle artificial reduction in attenuation value in the area anterior to the left lung.

Observing the effects additional projections have on the transmission images gives valuable insights into the truncation problem. It is also of interest to observe the effect of truncation on the reconstructed emission data. To measure the influence of additional projections on the emission data, the uniform disk was reconstructed using the

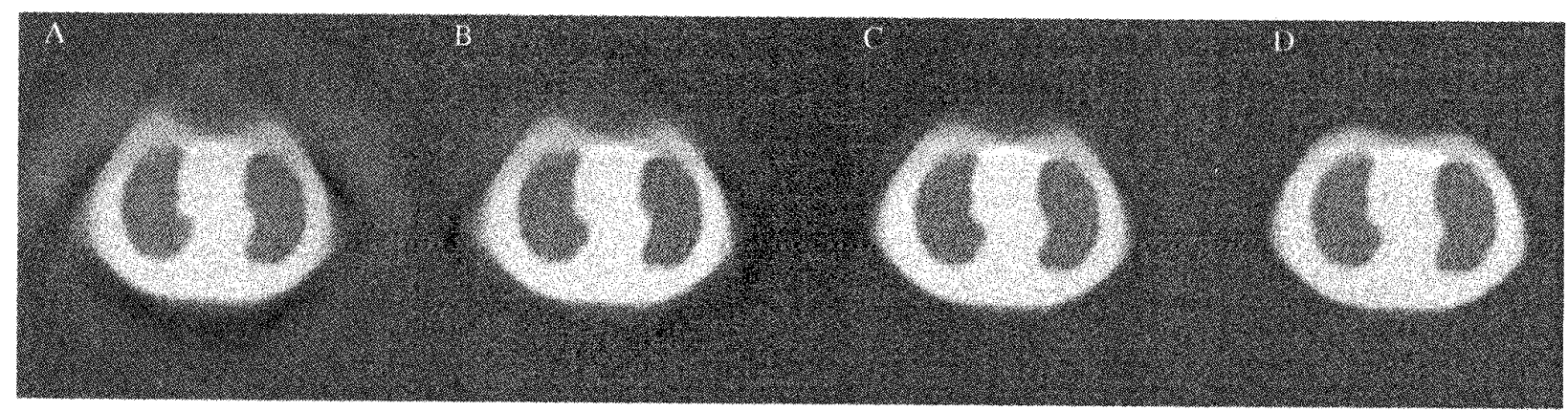

Fig. 5: Reconstructions of noise free parallel-beam truncated data (A: 128x128 grid, B: 110x110 grid, C: $90 \times 90$ grid, D: Contour) 


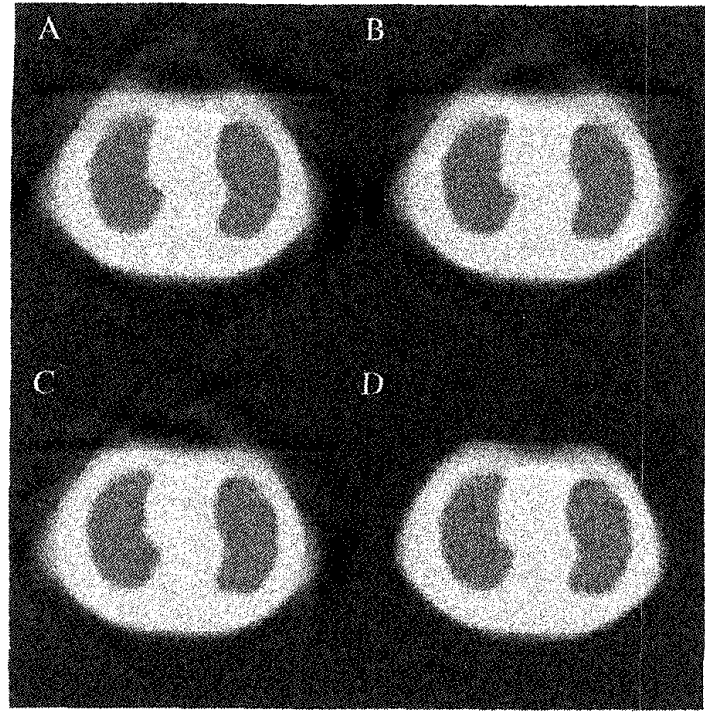

Fig. 7: $110 \times 110$ reconstructions of noise free parallel-beam truncated data (A: 1 proj., B: 2proj., C: 4 proj., D: Contour)

CT based attenuation map. This reconstruction with no truncation was taken as the reference image. The percentage difference between this reference image and the images reconstructed from the truncated attenuation maps, the truncated maps restored with the 1 to 4 additional projections, and the truncated map restored with knowledge of the contour were computed. We found an error of $6 \%$ on average for the case of a $128 \times 128$ grid and use of no additional information. This error was not uniform, reaching $10 \%$ in the upper part of the disk that was closer to the truncated area, and down to $1 \%$ at the bottom part. The average error was reduced by a factor of 2 by switching to a $110 \times 110$ grid. Completion with 4 projections yielded an average error of less than $1 \%$ with both grid sizes, equivalent to the use of the contour knowledge. This implies that the artifacts outside the body contour were sufficiently reduced by the completion technique to provide an accurate attenuation compensation.

Fig. 8 shows the images obtained from the measured data, using a $110 \times 110$ grid. Image 8 -A shows the FBP reconstruction of the complete data set. It can be compared to FWLSQ without use of additional information (8-B), with use of contour knowledge (8-C) and with use of 4 additional projections (8-D). The images are displayed postfiltered with a gaussian of fwhm 2 pixels, but the regularization was successful in ensuring a stable convergence. Fig. 9 shows the corresponding convergence curves computed inside the contour. The NRMS error was computed between the result for each iteration of FWLSQ and the FBP reconstruction of the untruncated transmission scan. To avoid measuring primarily noise differences

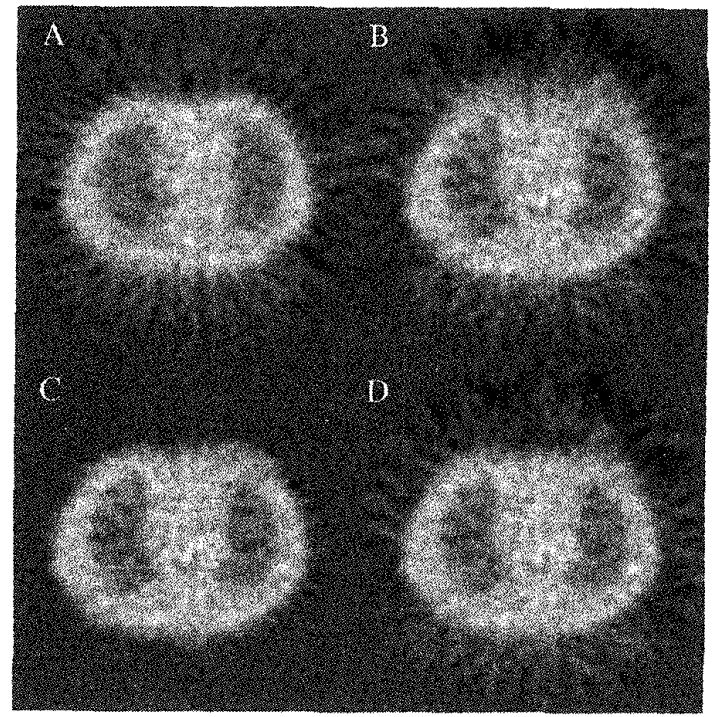

Fig. 8: $110 \times 110$ reconstructions of true parallel-beam transmission data. (A: Complete data, B: Truncated data, C: Contour, D: 4 proj.)

between FBP and FWLSQ, compared images were filtered by a gaussian of fwhm 4 pixels before computing the error. These measured results are consistent with the simulations. Equivalent restorations are seen between image 8-C (contour knowledge) and 8-D (limited completion), especially anterior to the lung. The subtle reduction in attenuation value of soft tissue anterior to the left lung, previously mentioned for the simulations, may still be present in figure $8 \mathrm{C}$ and $\mathrm{D}$, but is lost in the noise. Just as with the simulations, fig. 9 shows that the NRMS error inside the object is again slightly in favor of the completion technique.

In cardiac imaging, the attenuation maps that are usually obtained are very noisy, and the small residual errors present with both the proposed sinogram completion method and the contour knowledge method, are masked by the high noise. Therefore, it would seem preferable to optimize emission resolution by placing subjects as close to the collimator as possible, and correct for the resulting small transmission truncation by either the proposed sinogram completion method or by using knowledge of the body contour.

In the fan-beam case, the more extensive truncation results in a greater improvement in NRMS error by using the limited completion technique, compared to knowing the body contour. Fig. 10 shows the plot of the NRMS error for noise free simulated data when using FWLSQ with no additional information (128x128), contour knowledge and the completion schemes with $6,9,15$ and 30 projections per bed position. All completion schemes outperformed the contour knowledge inside the object, showing that more 


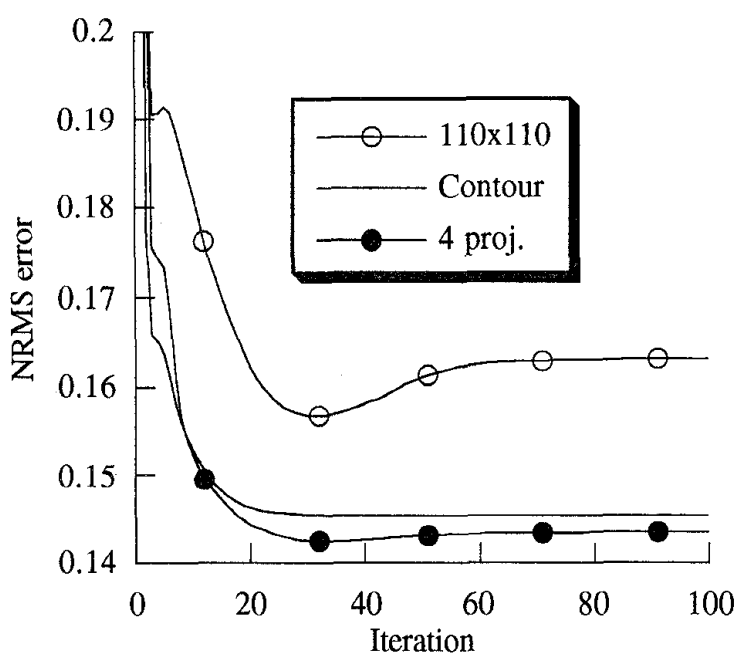

Fig. 9. Convergence of the FWLSQ algorithm for true parallel-beam transmission data with no additional information and a 110×110 grid, contour knowledge, and 4 additional projections and a $110 \times 110$ grid.

information is brought by the projections than by the contour knowledge with such a strong truncation, even when using a $128 \times 128$ grid.

Fig. 11 shows the corresponding transmission reconstructions. Image 11-A displays the reconstruction without additional information. The impact of contour knowledge is shown by image 11-B. Contour information results in a substantial improvement compared to figure $11 \mathrm{~A}$, but there is still significant inhomogeneity in the soft tissue and lungs. Recovery with limited completion is shown with images 11-C ( 6 projections per bed position) through $11-\mathrm{F}(30 \times 2)$. The information brought by those projections yielded a better uniformity of the truncated area and a recovery of the internal lung edges.

Errors in the attenuation corrected emission images were computed as in the parallel case, except that the CT image was used as both the transmission image (suitably scaled) and as the emission distribution (with activity proportional to attenuation value). The resulting error images are displayed on fig. 12 in correspondence with the attenuation maps shown in fig. 11. For a $128 \times 128$ grid and no additional information (image 12A) the error at the center can be quite high, due to the large truncation, with a whole area showing errors at the top of the gray scale, i.e. close to $20 \%$. Additional information reduced this error down to $2 \%$ (image 12B) with contour knowledge and $1 \%$ with any of the completion scheme (12C-F). More promising, is the reduction of the error beyond the fanbeam field of view. We do not consider the object boundaries where errors can be high because the emission

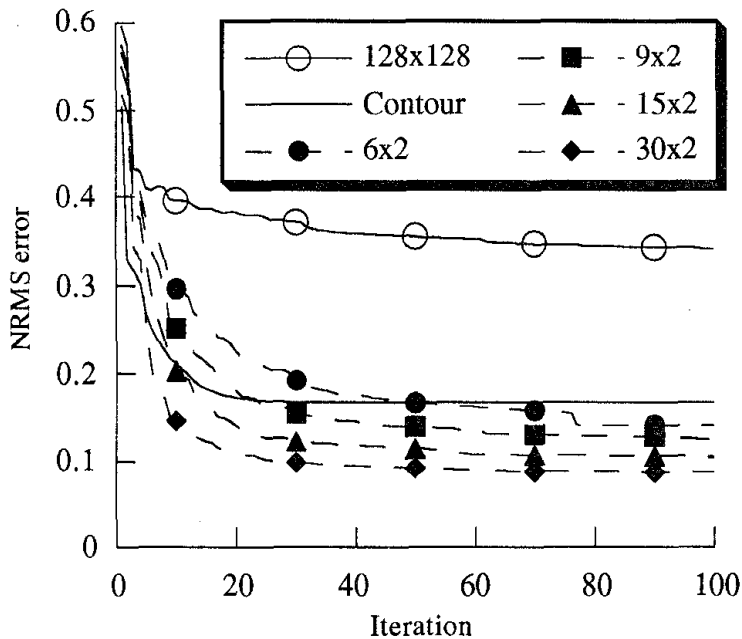

Fig. 10. Convergence of the FWLSQ algorithm for noise free fan-beam truncated data with no additional information, contour knowledge, and 4 completion schemes ( 6 projections $\times 2$ bed positions, $9 \times 2,15 \times 2,30 \times 2$ ).

values are small, as for a very small truncation at the bottom that we did not attempt to correct. With any of the completion schemes, the error was on average always lower than with the contour knowledge. As seen in fig. 11 , restoration based on contour knowledge did not provide sufficient uniformity around the edges of the lungs and the resulting emission errors in this area were comparatively higher. Using additional projections reduced those errors. We saw little difference between 15 additional projections per bed position and 30 (images $12 \mathrm{E}$ vs $12 \mathrm{~F}$ ), which is almost a complete set. The remaining error might be due to other causes (such as small errors in the fusion of the bed positions) rather than due to truncation itself.

In situations of low counts, noise could produce as large an error as truncation. However, even in these situations, it may be important to correct for truncation, since truncation produces a systematic error (e.g. a bias) rather than a random error. Such a correction can be accomplished with only a few additional projections. We also expect the completion technique to make attenuation correction significantly more accurate outside the ficld of view of the fan-beam collimator. The number of projections necessary to achieve an acceptable reduction in truncation error will depend to some extent on the noise in the data.

In all cases, the limited completion technique, even when based on very few additional projections, significantly improved the reconstruction of the attenuation maps. This improvement was often better than that achieved with knowledge of the contour. 


\section{CONCLUSION}

Acquisition of a few additional projections that sample the truncated area appears to obviate the need for body contour information. The limited completion technique makes use of readily available camera features, at least for a dual-headed gamma-camera, namely the ability to precisely set and record the bed position. Restoration of transmission maps by this method could be obtained in a clinically practical way, requiring very little increase in imaging time and no additional hardware (such as might be required to determine body contours).

Although three-headed systems equipped with fan-beam collimator do not usually offer sophisticated bed positioning, the newer generations of cameras are anticipated to provide detector motions. This should allow similar reduction in truncation artifacts similar to those obtained in the above simulations.

\section{REFERENCES}

[1] C. H. Tung, G. T. Gullberg, G. L. Zeng, P. E. Christian, F. L. Datz, and H. T. Morgan, "Nonuniform attenuation correction using simultaneous transmission and emission tomography," IEEE Trans. Nucl. Sci., vol. 39, pp. 1134-1143, 1992.

[2] P. Tan, D. L. Bailey, S. R. Meikle, S. Eberl, R. R. Fulton, and B. F. Hutton, "A scanning line source for simultaneous emission and transmission measurements in SPECT," J. Nucl. Med., vol. 34, pp. 1752-1760, 1993.

[3] E. P. Ficaro, J. A. Fessler, R. J. Ackerman, W. L. Rogers, J. R. Corbett, and M. Schwaiger, "Simultaneous transmissionemission Thallium-201 cardiac SPECT: Effect of attenuation correction on myocardial tracer distribution," J. Nucl. Med., vol. 36, pp. 921-931, 1995.

[4] G. K. Gregoriou, B. M. W. Tsui, and G. T. Gullberg, "Effect of truncated projections on defect detection in attenuationcompensated fan beam cardiac SPECT.," J. Nucl. Med., vol. 39, pp. 166-175, 1998.

[5] R. M. Lewitt, "Processing of incomplete measurement data in computed tomography," Med. Phys., vol. 6, pp. 412-417, 1979.

[6] K. Ogawa, M. Nakajima, and S. Yuta, "A reconstruction algorithm for truncated projections," IEEE Trans. Med. Imaging, vol. 3, pp. 33-40, 1984.

[7] S. Kawata and O. Nalcioglu, "Constrained iterative reconstruction by the conjugate gradient method," IEEE Trans. Med. Imaging, vol. 4, pp. 65-71, 1985.

[8] S. H. Manglos, "Truncation artifact suppression in conebeam radionuclide transmission CT using maximum likelihood techniques: evaluation with human subjects," Phys. Med. Biol., vol. 37, pp. 549-562, 1992.

[9] C. Riddell, A. Savi, M. C. Gilardi, and F. Fazio, "Frequency weighted least squares reconstruction of truncated transmission SPECT Data," IEEE Trans. Nucl. Sci., vol. 43, pp. 2292-2298, 1996.
[10] T. S. Pan, B. M. W. Tsui, and C. L. Byrne, "Choice of initial conditions in the ML reconstruction of fan-beam transmission with truncated projection data," IEEE Trans. Med. Imaging, vol. 16, pp. 426-438, 1997.

[11] D. J. Kadrmas, R. J. Jaszczak, J. W. McCormick, R. E. Coleman, and C. B. Lim, "Truncation artifact reduction in transmission CT for improved SPECT attenuation compensation," Phys. Med. Biol., vol. 40, pp. 1085-1104, 1995.

[12] D. R. Gilland, R. J. Jaszsczak, T. G. Turkington, and R. E. Coleman, "Comparison of transmission acquisition approaches for SPECT nonuniform attenuation compensation," IEEE Trans. Nucl. Sci., vol. 45, pp. 12441249, 1998.

[13] R. H. Huesman, G. T. Gullberg, W. L. Greenberg, and T. F. Budinger, "RECLBL Library Users Manual, Donner Algorithms for Reconstruction Tomography," Lawrence Berkeley Laboratory, Berkeley CA 1977.

[14] G. T. Gullberg, R. H. Huesman, J. A. Malko, N. J. Pelc, and T. F. Budinger, "An attenuated projector-backprojector for iterative SPECT reconstruction," Phys. Med. Biol., vol. 30, pp. 799-816, 1985.

[15] K. Lange and R. Carson, "EM reconstruction algorithms for emission and transmission tomography," $J$. Comput. Assist. Tomogr., vol. 8, pp. 306-316, 1984.

[16] J. A. Fessler, E. P. Ficaro, N. H. Clinthorne, and K. L. Lange, "Grouped-coordinate ascent algorithms for penalizedlikelihood transmission image reconstruction," IEEE Trans. Med. Imaging, vol. 16, pp. 166-175, 1997.

[17] A. P. Witkin, "Scale-space filtering," Proceedings of the Int. Joint Conf. Artificial Intelligence, Karlsruhe, Germany, pp. 1019-1022, 1983.

[18] J. J. Koenderink, "The structure of images," Biol. Cyber., vol. 50, pp. 363-370, 1984. 


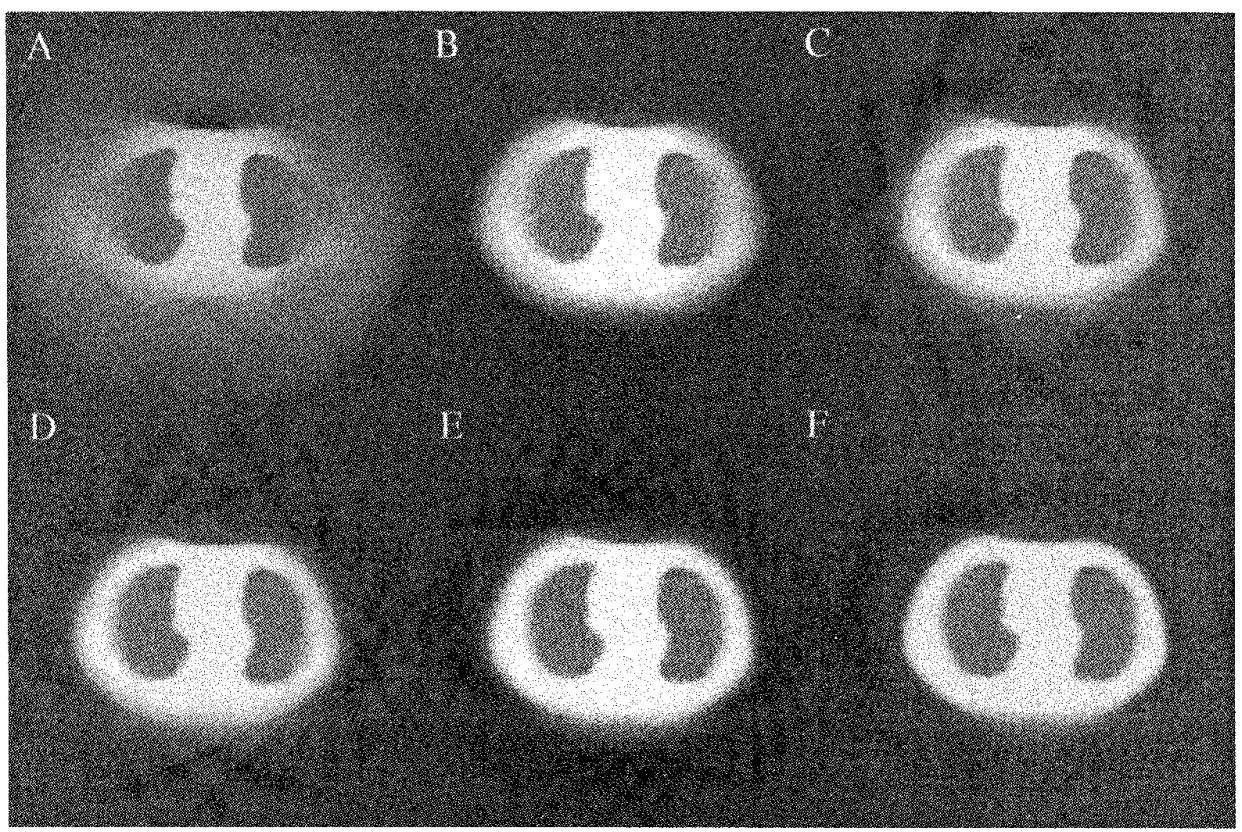

Fig. 11: Reconstructions of noise free fan-beam truncated data for 4 completion schemes (A: $128 \times 128$, B: Contour C: 6 proi. $\times 2$ bed positions, D: $9 \times 2$, E: $15 \times 2, F: 30 \times 2$ )

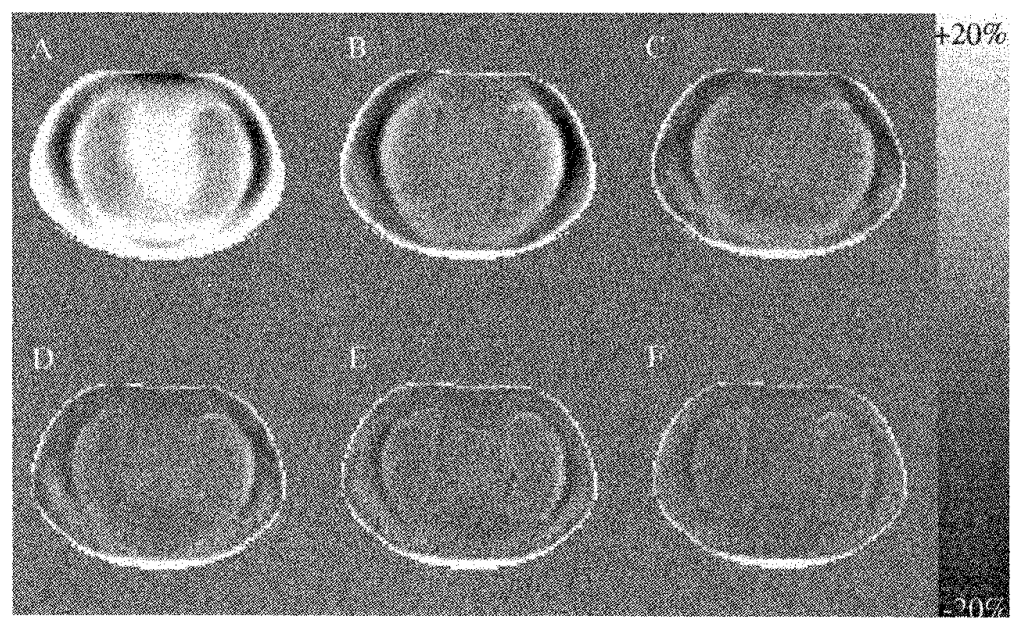

Fig. 12. Pixel \%error on the emission reconstruction with 8 different attenuation maps. Values are clipped at $\pm 20 \%$. (A: $128 \times 128$, B: Contour, C: 6 projections $\times 2$ bed positions, D: $9 \times 2$, E: $15 \times 2, \mathrm{~F}: 30 \times 2$ ) 